\title{
MODELO PARA PREDIÇÃO DE RESULTADOS DE COLHEITAS COM BASE EM MODELOS OCULTOS DE MARKOV
}

\author{
Jean Samarone Almeida Ferreira ${ }^{1}$, Ana Paula Lüdtke Ferreira ${ }^{1}$ e Naylor Bastiani Perez ${ }^{2}$ \\ ${ }^{l}$ Programa de Pós-graduação em Computação Aplicada, Universidade Federal do Pampa, Bagé/RS, Brasil \\ ${ }^{2}$ Embrapa Pecuária Sul, Bagé/RS, Brasil
}

\begin{abstract}
RESUMO
O gerenciamento adequado dos sistemas de produção agrícola pode garantir melhores resultados de produtividade, dado o tipo de colheita, condições climáticas, características do solo e outras variáveis agrícolas relevantes. A previsão dos resultados da colheita depende fortemente das características locais, e a literatura mostra que os fatores que afetam a produção variam no espaço e no tempo, exigindo que os sistemas para esse tipo de previsão possam alterar seu mecanismo de inferência ao longo do tempo. Este artigo propõe a modelagem de um sistema baseado em um modelo oculto de Markov para previsão da produtividade de culturas (a variável oculta), que utiliza dados relacionados às características do solo e condições climáticas (as variáveis visíveis) ao longo do tempo de plantio. A flexibilidade do modelo admite a adição de outras variáveis sem alteração de sua estrutura. Os resultados mostram que a variação temporal é capturada pelo modelo, que pode ser inicializado por uma abordagem de probabilidade frequentista ou bayesiana.
\end{abstract}

\section{PALAVRAS-CHAVE}

Predição de Colheita, Inferência Probabilística, Modelo Oculto de Markov

\section{INTRODUÇÃ̃O}

A produtividade das culturas agrícolas depende de uma combinação de fatores do ambiente de produção e das características genéticas das plantas (Franchini et al., 2016). O ambiente de produção envolve o solo, o clima local e o relevo, que podem ser alterados por atividades humanas de manejo. Contudo, ainda que as mesmas técnicas de plantio, manejo e colheita sejam realizadas, os resultados finais de produção apresentam variabilidade tanto espacial quanto temporal. A descoberta dos principais fatores associados a essa variabilidade permite estabelecer práticas de manejo voltadas à maximização dos resultados da colheita e à correta predição de resultados, dadas as condições presentes no ambiente de produção.

O levantamento das principais causas da variabilidade de produção é um tópico ativo de pesquisa nas Ciências Agrárias. Os resultados apresentados na literatura dependem fortemente dos dados disponíveis, que são usualmente específicos para cada cultura e local. Dessa forma, os resultados não podem ser extrapolados para outros lugares ou para diferentes tipos de culturas. Os achados referentes às causas primárias da variação da produtividade cobrem quase todas as variáveis agronômicas e meteorológicas: temperatura do ar (Franchini et al., 2016), déficit hídrico (Guedes-Filho, 2009), topografía (Kravchenko e Bullock, 2000), capacidade de troca catiônica (Usowicz e Lipiec, 2017) e assim sucessivamente. A dificuldade da análise das variáveis que intervêm nos resultados de produção parece ser intrínseca ao problema: Vieira e Gonzalez (2003) sugerem que os fatores que causam a variabilidade nos resultados de produção também variam com o tempo.

A previsão da produtividade de culturas é um problema relevante tanto do ponto de vista econômico quanto social, e mesmo uma solução parcial para o problema é desejável. Para ser útil, um modelo de previsão de rendimento de culturas deve lidar com a variabilidade espacial e temporal, além de fornecer informações ao produtor para que tome ações de gerenciamento ainda durante a estação de plantio. Os métodos empregados para definir as causas da variabilidade nos resultados de produção são, majoritariamente, baseados em técnicas de correlação e regressão, conforme apresentado em Cai et al. (2013) 
e Miller, Singer, Nilsen (1998) ou métodos geoestatísticos como discutido em Al-Omran et al. (2013), Mattioni, Schuch e Villela (2011) e Acosta et al. (2019). Ambas as abordagens possuem limitações: a primeira não captura causalidade ou relações não lineares (Murphy, 2012) e a segunda requer interpolação de pontos de dados que podem não representar as características do solo com precisão, além de não apresentar a capacidade de prover resultados ao longo do tempo.

Cadeias de Markov são modelos comumente utilizados em modelagem ecológica e ambiental (Paegelow e Olmedo, 2008), mas seu uso em modelos de predição agropecuários ainda é limitado. Diniz (1984) destacou a potencialidade de aplicação dos princípios Markovianos e dos recursos de tecnologia da informação aos estudos de transição e transformação da agricultura. Matis et al. (1985) e Matis, Birkett, Boudreaux (1989) apresentam formas diferentes de uso de cadeias de Markov para previsão de rendimento de culturas, caracterizando a generalidade e praticidade da metodologia que envolve esse tipo de modelo. Ainda segundo os autores, os modelos Markovianos caracterizam-se por suposições menos rigorosas e fornecem mais informações do que a regressão logística e, por consequência, são um procedimento de fácil adaptação entre aplicações similares. Thirunavukkarasu (2015) avaliou a tendência (aumento ou diminuição) da produção de cevada na Índia, com o uso de um modelo de Markov, que só depende das características do conjunto de dados passado, ao invés de um conjunto de pressupostos mais rigorosos. As análises de taxas de crescimento são amplamente empregadas para estudar o comportamento de diferentes produções agrícolas e costumam ser estimadas através de modelos paramétricos e séries temporais.

Este trabalho tem como objetivo verificar a aplicabilidade de modelos ocultos de Markov na predição e análise da variabilidade do resultado de colheitas. Cadeias de Markov modelam transições de probabilidade de um estado para outro, mas os agricultores estão mais interessados em como o ambiente afeta a produção, que uma estruturação em estados não é capaz de responder. A pergunta "qual é o rendimento de colheita mais provável desta plantação, tendo com base os dados atuais?" determina um problema de inferência de um conjunto de condições para um resultado final provável. Os modelos de ocultos de Markov separam as variáveis observáveis (fatores do ambiente de produção) das variáveis ocultas (resultados da produção), que não podem ser observadas diretamente. A contribuição deste trabalho concentra-se na construção de um modelo de previsão para resultados de colheita flexível e capaz de incorporar novas informações à medida que elas se tornam disponíveis, que é uma característica dos sistemas produtivos agropecuários.

A organização do trabalho é como se segue: a Seção 2 apresenta os materiais e métodos utilizados no desenvolvimento deste trabalho; a Seção 3 apresenta o modelo desenvolvido; a Seção 4 faz uma discussão sobre os resultados encontrados; finalmente, a Seção 5 conclui o trabalho com os achados relevantes.

\section{MATERIAL E MÉTODOS}

O trabalho é caracterizado como uma pesquisa aplicada, com revisão sistemática da literatura, coleta e pré-processamento de dados, construção do modelo de predição, implementação do modelo e posterior simulação. Os dados utilizados no teste do modelo são provenientes de duas fontes distintas: os dados meteorológicos são oriundos da base dados do Instituto Nacional de Meteorologia (INMET) e os dados de solo e de produção são provenientes de trabalhos de campo feitos pela equipe técnica da EMBRAPA Pecuária Sul. Os dados foram importados e pré-processados no Sistema de Informações Geográficas (QGIS, 2019) e representam uma base de 50 pontos para cada variável em cada uma das safras. Cada ponto representa uma coleta de dados (produtividade, solo, clima e altimetria).

As variáveis foram discretizadas conforme a bibliografia, mas para aquelas em que não havia uma definição de valores de classes, foi feita uma distribuição de frequências, onde o número de elementos pertencentes a cada classe é chamado de frequência de classe (Assis; Arruda; Pereira, 1996). Conforme orienta Spiegel e Stephens (2008), foi definida a amplitude total por meio do cálculo da diferença entre o maior e o menor valor do conjunto de dados. Após, a amplitude total foi dividida por três, de acordo com critério de mesmo tamanho de classe e número de classes pré-fixado, que definiu a amplitude de classe. $\mathrm{O}$ passo final foi determinar as frequências de classe ou intervalos de classe.

O algoritmo de inferência foi implementado na linguagem R (R Core Team, 2019), com os pacotes auxiliares TraMineR, gmodels, sf, tmap, readODS, gstat, sp, raster, rgdal. O pacote markovchain (Spedicato, 2019) foi usado para as funções mais básicas, como a geração das matrizes de transição e o pacote seqHMM (Helske e Helske 2019a, 2019b), para a organização dos dados, construção, análises e comparações de 
modelo. O pacote seqHMM inclui o algoritmo Viterbi e métodos para comparação de modelos. A comparação foi feita com o Bayesian Information Criterion (BIC), que permite comparar modelos com um número diferente de parâmetros. O BIC penaliza a complexidade do modelo a qual se refere ao número de parâmetros no modelo, como explica Robles et al. (2012).

Os resultados do modelo são apresentados como lista, gráficos sequenciais, gráficos percentuais, mapas espacializados em forma de pontos e mapas interpolados com o método Inverso da Potência das Distâncias (Inverse Distance Weighting - IDW), conforme Bivand, Pebesma e Gómez-Rubio (2008, p.193).

\section{O MODELO DE PREDIÇÃO}

Os modelos de Markov receberam esse nome em homenagem ao matemático russo Andrei Andreyevich Markov (1856-1922), que estabeleceu as bases da Teoria dos Processos Estocásticos. Um processo estocástico é uma cadeia probabilística de eventos ou situações em que eventos futuros da cadeia dependem dos anteriores (Taha, 2007). As cadeias de Markov são um tipo restrito de processo estocástico, em que o estado atual depende apenas do passado imediato, o que constitui um sistema de transição de estados. Uma transição do estado A para o estado B representa a probabilidade condicional $\mathrm{p}(\mathrm{B} \mid \mathrm{A})$, ou a probabilidade do sistema passar para o estado B, dado que atualmente está no estado A. Um modelo oculto de Markov (Fink, 2008; Jurafsky e Martin, 2018; Rabiner e Juang, 1986) é uma tupla $M=(S, V, A, B, \pi)$, onde $S$ é um conjunto finito de estados; $\mathrm{V}$ é um conjunto de valores observáveis; A é uma matriz de transição de probabilidade em que cada elemento $a_{i j}$ de A representa a probabilidade condicional p $\left(S_{j} \mid S_{i}\right)$, ou a probabilidade do sistema $M$ passar ao estado $S_{j}$ dado que o estado atual é $S_{\mathrm{i}}$; B é a matriz de probabilidade de observação em que cada elemento $b_{j k}$ em $B$ corresponde à probabilidade condicional $p\left(V_{k} \mid S_{j}\right)$, ou à probabilidade dos valores observados ser $V_{k}$, dado que o sistema $M$ está no estado $S_{j}$. $O$ modelo $\mathrm{M}=(\mathrm{S}, \mathrm{V}, \mathrm{A}, \mathrm{B}, \pi)$ às vezes é escrito como $\mathrm{M}=(\mathrm{A}, \mathrm{B}, \pi)$, pois o primeiro pode ser inferido pelo último.

Os modelos ocultos de Markov apresentam três problemas associados: i) o problema da avaliação (likelihood ou probability evaluation); ii) o problema da decodificação (decoding ou optimal state sequence); e iii) o problema do aprendizado (learning ou parameter estimation) (Rabiner e Juang, 1986). O problema de interesse, no contexto deste trabalho, é o segundo, que permite que dados meteorológicos, gerenciais e agronômicos localmente coletados possam ser inseridos no modelo para prever o rendimento futuro mais provável da colheita. O problema de decodificação consiste em encontrar o resultado para a seguinte pergunta: dado um modelo $\mathrm{M}$ e uma sequência de observações $\mathrm{O}=\mathrm{o}_{1}, \mathrm{O}_{2}, \ldots, \mathrm{O}_{\mathrm{n}}$, qual é a sequência mais provável de estados ocultos obtidos? A solução eficiente deste problema é dada pelo algoritmo de Viterbi (Viterbi, 1967), que apresenta uma solução ótima recursiva para o problema de estimar a sequência de estados ocultos de um processo de Markov (Forney, 1973).

A estimativa feita por meio de modelos ocultos de Markov necessita de um conjunto de probabilidades iniciais, uma matriz de transição de probabilidades (parte oculta) e uma matriz de probabilidades de observação (ou emissão). Os valores iniciais também podem ser usados para definir restrições à estrutura do modelo. As probabilidades iniciais fornecem a probabilidade de iniciar em um dado estado oculto. No presente caso, formam um vetor com a probabilidade do resultado de safra (alta, baixa e média), em cada posição do vetor. Uma probabilidade com valor maior significa uma maior chance de iniciar naquele estado. No modelo proposto, cada estado representa um resultado da colheita em cada ponto, categorizado como um entre três possíveis resultados de produção: alta, baixa e média, o que caracteriza a parte oculta do modelo, como mostrado na Figura 1.

A matriz de transição de estados é calculada a partir dos dados de produção já existentes. O cálculo das probabilidades foi feito a partir dos dados de produção, referentes a quatro (4) safras distintas, dentro das áreas demarcadas com dados de amostra do solo. Como esses dados somente estão disponíveis em 50 pontos da área, todos os dados de entrada do modelo foram extraídos das regiões correspondentes. Assim, tem-se uma matriz de 50 linhas e 4 colunas, com os dados de produção de cada ponto, em cada uma das safras. A matriz de transição resultante, para os dados de produção da área objeto de estudo é dada na primeira matriz da Figura 2. Cada intersecção de linha e coluna informa a probabilidade condicional de um resultado de produção, dado o resultado de produção do ano anterior. A soma dos valores em cada linha da matriz é 1, o que caracteriza uma distribuição probabilística discreta. 


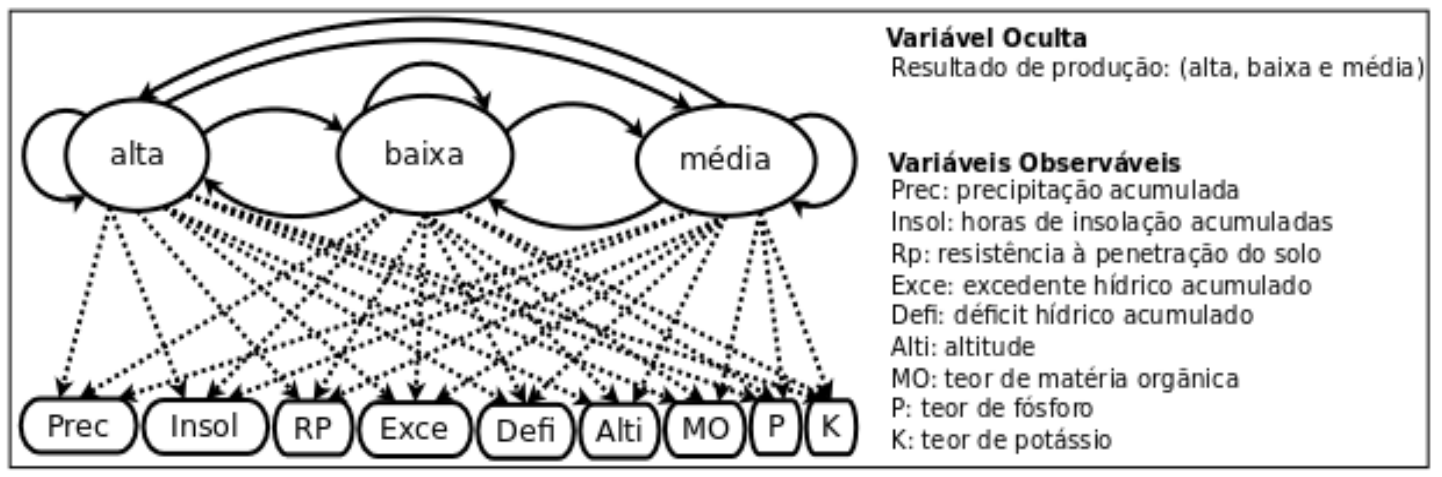

Figura 1. Modelo oculto de Markov para predição de colheita

$$
M=\left[\begin{array}{ccc}
p_{a \mid a} & p_{a \mid b} & p_{a \mid m} \\
p_{b \mid a} & p_{b \mid b} & p_{b \mid m} \\
p_{m \mid a} & p_{m \mid b} & p_{m \mid m}
\end{array}\right] M=\left[\begin{array}{cccc}
p_{a \mid O a} & p_{a \mid O b} & \cdots & p_{a \mid O n} \\
p_{b \mid O a} & p_{b \mid O b} & \cdots & p_{b \mid O n} \\
p_{m \mid O a} & p_{m \mid O b} & \cdots & p_{m \mid O n}
\end{array}\right]
$$

Figura 2. Matriz de transição de probabilidades (parte oculta) e matriz de probabilidades de observação (ou emissão). As siglas $(\mathrm{Pa}|\mathrm{a}, \mathrm{Pa}| \mathrm{b}, \mathrm{Pa}|\mathrm{m}, \mathrm{Pb}| \mathrm{a}, \mathrm{Pb} \mid \mathrm{b}$ e $\mathrm{Pb} \mid \mathrm{m})$, representam a probabilidade condicional de um resultado de produção, dado o resultado de produção do ano anterior. As siglas $(\mathrm{Pa}|\mathrm{Oa}, \mathrm{Pa}| \mathrm{Ob}, \mathrm{Pb} \mid \mathrm{Oa}$ e $\mathrm{Pb} \mid \mathrm{Ob})$ representam a relação entre a variável oculta (peso produzido) e as variáveis observáveis

As probabilidades restantes do modelo dizem respeito à relação existente entre a variável oculta (peso produzido) e as variáveis observáveis: precipitação acumulada (Prec), horas de insolação acumuladas (Insol), resistência à penetração do solo (RP), excedente hídrico acumulado (Exce), déficit hídrico acumulado (Defi), altitude (Alti), teor de matéria orgânica (MO), teor de fósforo $(\mathrm{P})$ e teor de potássio $(\mathrm{K})$, conforme a segunda matriz na Figura 2 e representadas na parte inferior da Figura 1.

O modelo e o algoritmo propostos são flexíveis e permitem que com poucas alterações no código possase alterar o número de variáveis observáveis, bem como os estados possíveis para cada variável. Também é possível organizar o modelo em qualquer intervalo de tempo desejado, como por exemplo: safra, dia, fase, mês ou outro intervalo desejado qualquer. Para o teste do algoritmo foram utilizados dados reais de produtividade que representam a variável oculta (resultado de produção) em conjunto com dados reais e simulados de clima, compactação do solo e valores altimétricos, que representam as variáveis observáveis. Devido à restrição do conjunto de dados (somente 4 safras) e à ausência de dados em algumas variáveis houve a necessidade de gerar dados simulados. Pela característica geográfica dos dados (georreferenciados), eles foram pré-processados em Sistema de Informações Geográficas (SIG) antes de sua utilização como entrada no algoritmo. $\mathrm{O}$ algoritmo foi desenvolvido na linguagem $\mathrm{R}$, pois vários pacotes implementam funcionalidades relacionadas aos modelos de Markov.

\section{RESULTADOS OBTIDOS}

As probabilidades condicionais e marginais foram calculadas a partir dos dados das safras disponíveis, onde duas delas tiveram resultados médios de produção, uma teve um valor alto e outra um valor baixo. Na Figura 3 é apresentado o modelo oculto de Markov gerado, como um grafo dirigido com nove variáveis. Os círculos/vértices representam os três estados ocultos (peso alto, peso baixo e peso médio) e os setores/partições as probabilidades de emissão das combinações dos estados observados. As setas/arestas mostram as probabilidades de transição; as probabilidades iniciais estão abaixo dos vértices. Estados observados com probabilidade menor do que $1 \%$ estão agrupados em um único setor (cor branca). As probabilidades estimadas como zero não são mostradas e, para fins de legibilidade, as probabilidades de transição foram arredondadas. 


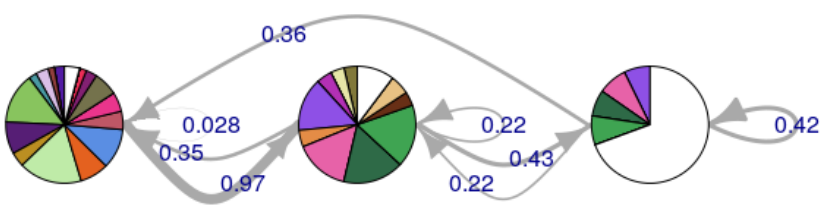

0.25

0.25

Peso Alto

Peso Baixo

0.5

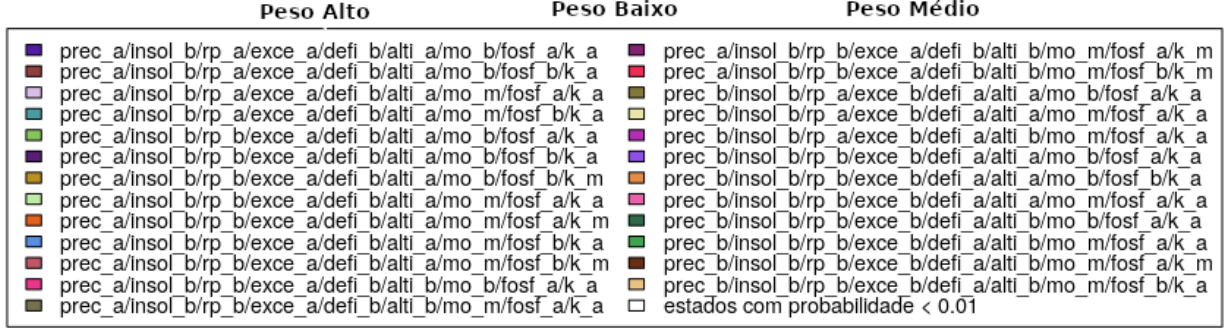

Figura 3. Grafo dirigido do modelo oculto de Markov

Na Figura 4 é apresentado, de forma sequencial e percentual, o caminho mais provável. O resultado aponta uma primeira safra com produtividade alta $(48 \%)$, baixa $(0 \%)$ e média $(52 \%)$. A segunda safra com produtividade alta (12\%), baixa (50\%) e média (38\%). A terceira safra com produtividade alta $(80 \%)$, baixa $(12 \%)$ e média $(8 \%)$. A quarta safra com produtividade alta $(6 \%)$, baixa $(80 \%)$ e média $(14 \%)$. O gráfico com a sequência de dados (50 observações) e distribuição percentual permite avaliar a variabilidade espacial da produtividade dentro da área de estudo e também ao longo do tempo.
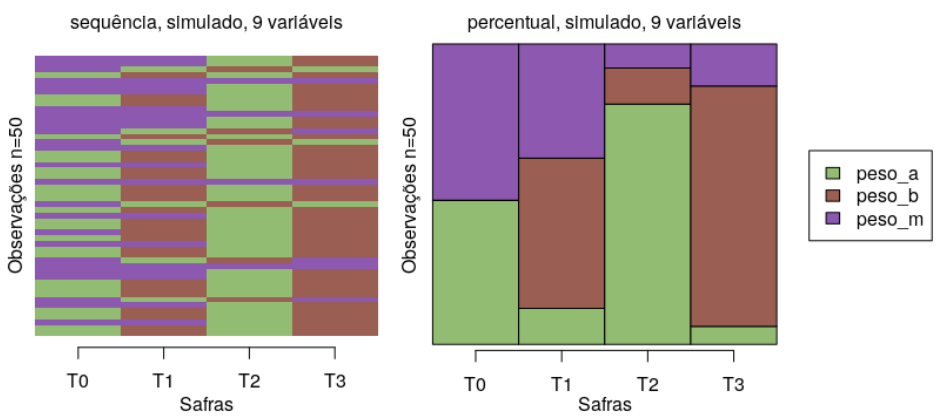

Figura 4. Caminho mais provável em formato sequencial e percentual

No extrato de código abaixo é apresentada a sequência de estados mais provável em cada ponto (somente os três primeiros, de 50 pontos). Essa mesma sequência foi utilizada para a construção dos mapas mostrados na Figura 5.

1 peso_m - peso_m - peso_a - peso_b

2 peso_m - peso_m - peso_a - peso_b

3 peso_m - peso_a - peso_b - peso_a

Na Figura 5 é apresentada a produtividade predita interpolada (IDW) para as quatro safras (T0, T1, T2 e T3), no modelo completo (nove variáveis). O mapa, além de identificar o local onde foram coletados os dados de peso, amostras de solo e resistência à penetração, permite obter uma estimativa com valores intermediários entre dois ou mais pontos existentes.

Os resultados apontam para a necessidade de continuidade do estudo através da ampliação do conjunto de dados, o que evitaria a duplicação e simulação de dados faltantes. Uma qualificação na discretização por meio de técnicas mais apuradas e participação efetiva de um especialista. O algoritmo necessita de uma interface gráfica e integração com SIG, de forma que permita a geração de cenários diversos. O cálculo do caminho mais provável permite que se tenha a predição probabilística de forma rápida e em formato inteligível, o qual foi utilizado para a geração de mapas. 


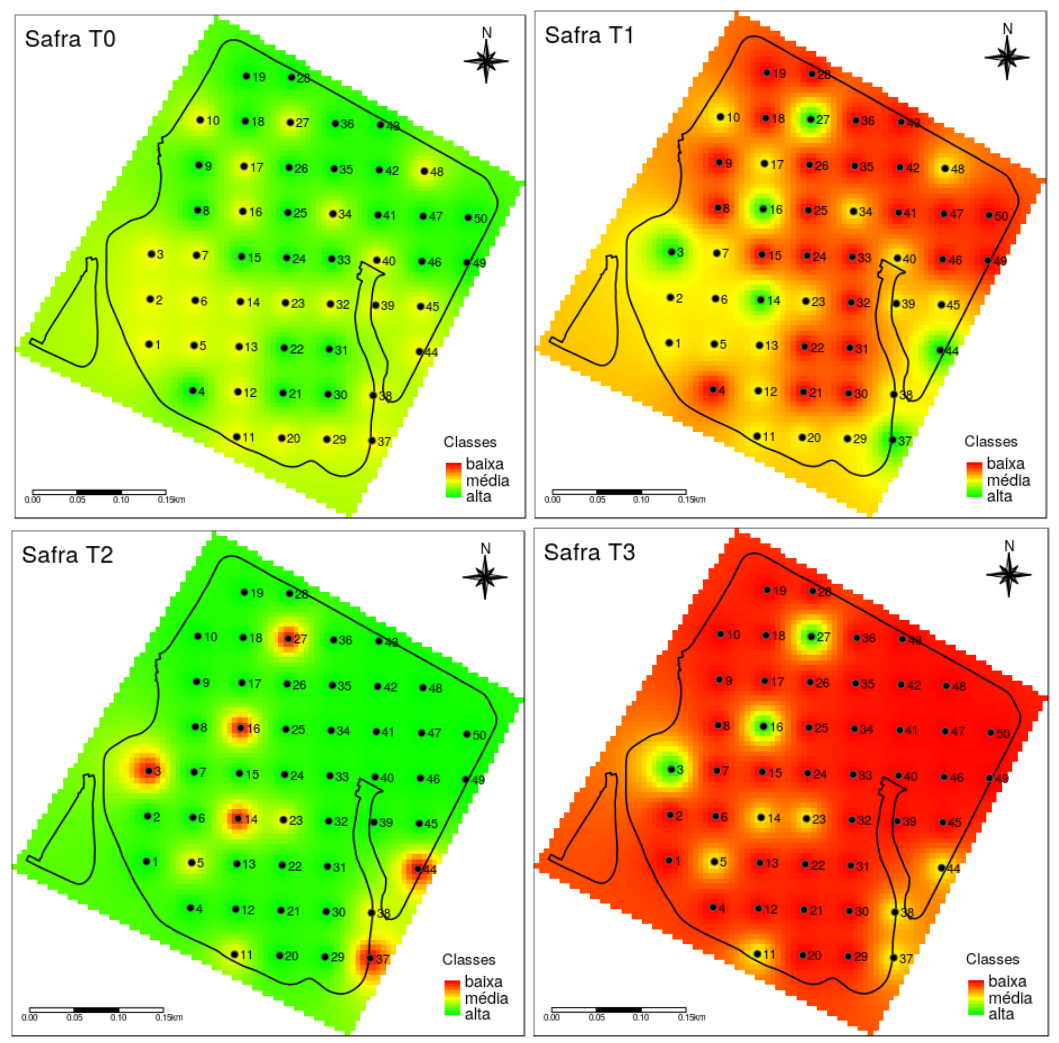

Figura 5. Mapas gerados a partir sequência de estados mais provável e interpolados para as safras (T0, T1, T2 e T3)

Os fenômenos que ocorrem dentro de cada fase fenológica são elementos importantes para o entendimento da variabilidade na produtividade. Pretende-se estender o modelo, para que o tempo seja dividido em fases de uma safra. Para um modelo desse nível é importante a coleta de dados que façam referência a cada uma das fases de interesse, o que não estava disponível quando da realização deste trabalho.

A partir do conjunto de funções em R, desenvolvidas neste trabalho, pode-se implementar um módulo que permita a integração com um SIG. Nesse caso, todo o processo de entrada de dados, modelagem e resultados seria feito dentro do SIG.

\section{CONCLUSÃO}

A variabilidade espacial da produtividade é uma questão complexa e várias abordagens têm sido utilizadas para tentar resolver ou atenuar seus efeitos no resultado final de uma ou de um conjunto de safras, bem como em locais específicos dentro de uma área. Os estudos comumente realizados focam somente na relação entre as variáveis em um determinado instante, e deixam de lado a evolução do processo ao longo do tempo. Os modelos ocultos de Markov estabelecem um relacionamento probabilístico entre os estados ocultos e as observações feitas ao longo do processo, que evolui no tempo por meio das transições entre seus estados. No modelo proposto a única exigência é que o estado futuro dependa apenas do estado presente e não dos estados passados, sem a necessidade de atender suposições mais rigorosas sobre relações entre variáveis meteorológicas, variáveis agronômicas e ações de manejo, como em outros tipos de modelo. Essa restrição pode ser considerada forte para um modelo de cultura, em que os estados futuros dependem de mais de um estado passado. Contudo, se dados acumulados forem usados, a restrição passa a ser incorporada ao modelo, que passa a comportar-se como um modelo de Markov.

O modelo mostrou-se adequado para predizer a produtividade ao longo das safras, mas a estimativa da variabilidade dentro de uma determinada área é mais sensível à disponibilidade e à discretização dos dados de entrada. Para que o resultado do modelo seja confiável é necessário um conjunto maior de dados. Em um 
conjunto pequeno de dados, como o utilizado neste trabalho, as probabilidades podem ser estimadas com uma abordagem bayesiana, em oposição à abordagem frequentista, que requer um número maior de observações.

\section{REFERÊNCIAS}

Acosta, J. J. B. et al., 2019. Variabilidade espacial da produtividade, perdas na colheita e lucratividade da cultura de soja. Revista Agrogeoambiental, IFSULDEMINAS, v. 10, p. 27-46, ISSN 2316-1817.

Al-Omran, A. et al., 2013. Spatial variability for some properties of the wastewater irrigated soils. Journal of the Saudi Society of Agricultural Sciences, v. 12, n. 2, p. 167 - 175. ISSN 1658-077X.

Assis, F. N.; Arruda, H. V. de; Pereira, A. R., 1996. Aplicações de Estatística à Climatologia - Teoria e Prática. 8th. ed. Pelotas: Editora Universitária UFPEL, $161 \mathrm{p}$.

Bivand, R. S.; Pebesma, E.; Gómez-Rubio, V., 2008. Applied Spatial Data Analysis with R. New York, NY: Springer, $374 \mathrm{p}$.

Cai, R. et al., 2013. Using a climate index to measure crop yield response. Journal of Agricultural and Applied Economics, v. 45, n. 4, p. 18.

Diniz, J. A. F., 1984. Geografia da Agricultura. São Paulo: Difel. 278 p.

Fink, G. A., 2008. Markov Models for Pattern Recognition - From Theory to Applications. 8th. ed. Londres: Springer-Verlag, $248 \mathrm{p}$.

Forney, G. D., 1973. The viterbi algorithm. Proceedings of IEEE, v. 61, n. 3, p. 268-278.

Franchini, J. C. et al, 2016. Variabilidade espacial e temporal da produção de soja no Paraná e definição de ambientes de produção. Embrapa Soja, Londrina, Brasil. ISSN 2176-2937.

Guedes-Filho, O., 2009. Variabilidade espacial e temporal de mapas de colheita e atributos do solo em um sistema de semeadura direta. Master's Thesis. Instituto Agronômico, Campinas, São Paulo. 114 p.

Helske, J.; Helske, S., 2019. seqHMM: Mixture hidden Markov models for social sequence data and other multivariate, multichannel categorical time series. $\mathrm{R}$ package version 1.0.14. Disponível em: https://www.rdocumentation.org/packages/seqHMM/versions/1.0.14.

Helske, S.; Helske, J., 2019. Mixture hidden Markov models for sequence data: The seqHMM package in R. Journal of Statistical Software, v. 88, n. 3, p. 1-32.

Jurafsky, D.; Martin, J. H., 2018. Speech and Language Processing. Disponível em: <https://web.stanford.edu/ jurafsky/slp3/ed3book.pdf>. Acessado em: 12 março 2019

Kravchenko, A. N., and Bullock, D. G., 2000. Correlation of corn and soybean grain yield with topography and soil properties. Agronomy Journal, American Society of Agronomy, v. 92, n. 1, p. 75-8. ISSN 0002-1962.

Matis, J. et al., 1985. A markov chain approach to crop yield forecasting. Agricultural Systems, v. 18, n. 3, p. $171-187$. ISSN 0308-521X.

Matis, J. H.; Birkett, T.; Boudreaux, D., 1989. An application of the markov chain approach to forecasting cotton yields from surveys. Agricultural Systems, v. 29, n. 4, p.357-370.

Mattioni, N. M.; Schuch, L. O. B.; Villela, F. A., 2011. Variabilidade espacial da produtividade e da qualidade das sementes de soja em um campo de produção. Revista Brasileira de Sementes, scielo, v. 33, p. 608 - 615, ISSN 01013122.

Miller, M. P.; Singer, M. J.; Nielsen, D. R., 1998. Spatial variability of wheat yield and soil properties on complex hill. Soil Science Society of American Journal, v. 52, p.1133-1141. ISSN 0361-5995.

Murphy, K. P., 2012. Machine Learning - A Probabilistic Perspective. Cambridge, USA: MIT Press. 1067 p.

Paegelow, M. and Olmedo, M. T. C. 2008. Modelling Environmental Dynamics - Advances in Geomatic Solutions. Berlin Heidelberg: Springer-Verlag. (Environmental Science).

QGIS. QGIS Geographic Information System. 2019. Disponível em: <http://qgis.osgeo.org>. Acessado em: 25 março 2019

Rabiner, L. R. and Juang, B. H. 1986. An introduction to hidden Markov models. IEEE ASSP Magazine 3:4-16.

Robles, B. et al., 2012. Methods to choose the best hidden markov model topology for improving maintenance policy. In: International Conference of Modeling, Optimization and Simulation. Bordeaux, France.

R Core Team., 2019. R: A Language and Environment for Statistical Computing.Vienna, Austria: R Foundation for Statistical Computing. Disponível em:<http://www.R-project.org/>. Acessado em: 15 janeiro 2019.

Spedicato, G. A., 2019. Package markovchain. Vienna, Austria, Disponível em:<https://cran.rproject.org/web/packages/markovchain/markovchain.pdf>. Acessado em:15 janeiro 2019. 
Spiegel, M. R.; Stephens, L. J., 2008. Theory and Problems of Statistics. 4th. ed. NewYork: McGRAW-HILL, 577 p. Taha, H. A. 2007. Operations Research: An Introduction. 8th ed. Upper Saddle River, New Jersey: Pearson Prentice Hall. $840 \mathrm{p}$.

Thirunavukkarasu, M., 2015. Stochastic modeling in agricultural production. 209 p. Tese (Doutorado) — Manonmaniam Sundaranar University, Tirunelveli, Tamilnadu, Índia.

Vieira, S. R.; Gonzales, A. P., 2003. Analysis of the spatial variability of crop yield and soil properties in small agricultural plots. Bragantia, scielo, v. 62, p. 127 - 138, 00 2003.ISSN 0006-8705.

Viterbi, A. J., 1967. Error bounds for convolutional codes and an asymptotically optimum decoding algorithm. IEEE Transactions on Information Theory 13(2):260-269.

Usowicz, B.; Lipiec, J., 2017. Spatial variability of soil properties and cereal yield in a cultivated field on sandy soil. Soil and Tillage Research, v. 174, p. 241 - 250. ISSN 0167-1987. 